\title{
LE STATUT DU TERRITOIRE ET LE PACTE DE PROGRÈS
}

C Vernaudon*

Pour pouvoir traiter ce sujet, il faut au préalable avoir défini les termes qui le composent.

Or, si le statut du Territoire est un texte clairement identifié, il n'en va pas de même pour le Pacte de progrès.

Ce terme de Pacte de progrès est en effet abondamment utilisé depuis deux ans sans que son contenu exact n'ait été clairement identifié ce qui amène les différents interlocuteurs qui le cite à lui donner des définitions parfois très distinctes les unes des autres.

Ainsi, sous un angle politique, le Pacte de progrès est devenu aujourd'hui: le seul espoir de salut pour la Polynésie française pour les uns et il serait déjà mort-né pour les autres.

Pour ma part, je vous propose de structurer mon intervention autour de trois définitions possibles du Pacte de progrès car à mes yeux le Pacte de progrès fût en premier lieu une démarche avant d'être l'expression d'un projet de société et enfin sera ou ne sera pas la concrétisation d'une nouvelle organisation économique, sociale et culturelle sur le Territoire en fonction de ce que les hommes voudront en faire.

\section{Le pacte de progrès: Une démarche}

En écoutant hier l'exposé de Mr Regnault sur la vie politique du Territoire de 1984 à 1994 et sur le caractère animé et souvent très conflictuel de cette dernière, il m'a semblé qu'avait échappé à l'analyse de Mr Regnault une courte parenthèse au cours de laquelle une quasi union sacrée avait pu être observée dans notre petit monde politico-social.

Je situe pour ma part cette parenthèse entre avril 1992 et janvier 1993, période d'élaboration de la Charte du développement et du Pacte de progrès.

Conseiller au commerce exterieur pour la Polynésie Française et membre du Conseil économique et social à Paris. 
De fait, la Polynésie française a connu durant cette période une activité particulièrement intense de réflexion et de remise en cause de la part de l'ensemble des forces sociales et politiques.

Se sont ainsi succédés les travaux de l'IHEDN (Institut des Hautes Etudes de la Défense Nationale), les ateliers généraux de la Charte du développement, les travaux du CESC (Conseil Economique, Social et Culturel) sur le thème d'autosaisine: "Quel choix pour l'avenir de la Polynésie" enfin, les travaux d'élaboration des propositions de la délégation polynésienne pour le Pacte de progrès.

L'ensemble de ces réflexions et travaux ont constitué à la fois un auto-diagnostic et une auto-critique en profondeur de la société polynésienne par elle-même pour ensuite jeter les bases d'une définition d'un projet de société pour la Polynésie française pour la décennie à venir.

Je vous invite à consulter les documents produits tant par l'IHEDN que le CESC et surtout la Charte du développement pour constater que notre auto-critique fut sans aucune complaisance emportant simultanément dans ses flots les politiques, l'Etat, les syndicalistes, le patronat, les églises, les medias et en définitive chaque citoyen polynésien qui avait profité du système depuis 25 ans sans réagir et surtout sans penser à l'avenir de ses enfants.

Mais ces différents travaux ont également constitué un diagnostic particulièrement clair des forces et faiblesses de notre économie et de notre société à partir duquel ont été proposés des axes de développement et de réformes.

Mais me direz-vous, quel rapport avec le statut et avec le Pacte de progrès?

Il se trouve que l'aboutissement de ce vaste chantier de réflexion fut la production en décembre 1992 d'un document intitulé Propositions de la délégation polynésienne pour le Pacte de progrès. Comme nous le verrons au chapître suivant, ce document constitue un véritable projet de société pour la Polynésie française et s'il fallait trouver un texte qui soit le Pacte de progrès, ce serait à mon avis plutôt celui-là que la loi d'orientation ou le contrat de développement comme cela a pu être dit ultérieurement.

La caractéristique fondamentale de ce document est qu'il a été élaboré en Polynésie et par les polynésiens dans le cadre d'une concertation sans précédent qu'ont salué successivement les ministres des DOM-TOM, Messieurs Le Pensec et Perben ainsi que le Premier Ministre de la République, Mr Balladur.

Le Conseil Economique, Social et Culturel a été largement associé à son élaboration et l'a soutenu à l'unanimité. Mais il faut surtout rappeler que ces propositions pour le Pacte de progrès ont été adoptées à la quasi-unanimité par les conseillers territoriaux donc majorité et opposition autonomiste confondues. 
Seule l'opposition indépendantiste s'est abstenue bien qu'elle ait fait savoir qu'elle aurait pu soutenir ce texte s'il avait intégré un scrutin d'auto-détermination à échéance de 10 ans dans ses recommandations.

Pour la première fois et très exactement dans l'esprit et dans la lettre du texte du statut d'Autonomie interne, les polynésiens avaient donc défini entre eux ce que devait être leur nouvelle économie, leur nouvelle société et, aussi nous allons le voir, le cadre statutaire souhaité pour les 10 ans à venir.

Voilà en quoi, le Pacte de progrès fût en premier lieu une démarche respectant le fondement de l'esprit du statut qui est qu'il appartient fondamentalement à la population polynésienne et à ses élus de définir l'environnement social, économique et culturel dans lequel elle souhaite inscrire son développement.

On peut s'interroger sur les raisons qui ont permis d'obtenir l'union sacrée politique qui fît qu'aussi bien Messieurs Gaston Flosse et Jean Juventin que Messieurs Alexandre Leontieff et Emile Vernaudon ou encore Daniel Millaud et Raymond Desclaux devaient soutenir ce texte à l'Assemblée Territoriale et ensuite d'une même voix devant le Ministre des DOM-TOM, Monsieur Louis Le Pensec quelque peu surpris à l'évidence de trouver pour la première fois de son histoire une représentation politique de la Polynésie française unanime à Paris pour défendre un projet commun.

Sans aucun doute, l'importance des enjeux pour le Territoire et le soutien unanime et puissant des forces vives apporté à la démarche furent-ils des éléments déterminants dans cette cohésion.

Je pense qu'il s'y est ajouté un facteur supplémentaire extrêmement bénéfique qui était alors lié à la constitution politique de la délégation polynésienne.

En effet, cette dernière comprenait de droit, le Président du Gouvernement du Territoire, le Président de l'Assemblée Territoriale, les deux Députés, le Sénateur, le Conseiller économique et social et le Président du CESC.

Or, il se trouve qu'à cette période là, les postes de présidents des institutions locales et de parlementaires étaient très équitablement partagés entre les différentes composantes du paysage politique polynésien à l'exclusion du parti indépendantiste de Monsieur Temaru.

Il y avait donc de facto un large partage du pouvoir politique qui fût je le pense déterminant pour assurer le succès de la première phase de la démarche, celle de l'élaboration consensuelle de notre projet de société.

\section{Le pacte de progrès: Un projet de société}

L'élément majeur du diagnostic du Pacte de progrès était que la Polynésie française avait bénéficié pendant 25 ans d'un schéma de développement économique basé sur les transferts 
du CEP comme l'a rappelé hier Monsieur Bernard Poirine, mais plus encore comme l'ensemble des autres DOM-TOM sur le système d'économie de rente créé par les transferts civils de l'Etat prenant principalement la forme de rémunérations de fonctionnaires avec application d'un fort coefficient multiplicateur par rapport à la métropole.

Outre ses effets très pervers constatés dans tout l'outre-mer français, ce système ne pouvait plus du tout se perpétuer en Polynésie française dès lors que l'un de ses moteurs majeur, le CEP, était potentiellement remis en cause.

L'économie de la rente avait, par ailleurs, permis grâce à de très importants mécanismes de redistribution (subvention au coprah, régime de protection sociale en milieu rural, embauches de personnels non qualifiés dans les administrations territoriales et les communes...) d'assurer un emploi et des revenus décents à une très large fraction de la population jusqu'au milieu de la décénnie 80 .

Mais la combinaison d'une forte croissance démographique et d'un net ralentissement de la croissance économique induit par une nette réduction du taux de croissance annuel des transferts de l'Etat conduisait depuis déjà près de 10 ans à un développement très rapide du chômage et de l'exclusion qui touche désormais environ $20 \%$ de la population.

Les schémas ci-après résument, en conséquence, les principaux constats établis au début des annés 90 (voir par ailleurs les tableaux de référence de la Charte du développement) et les principaux objectifs fixés en $\mathbf{1 0}$ ans dans le cadre du Pacte de progrès. 


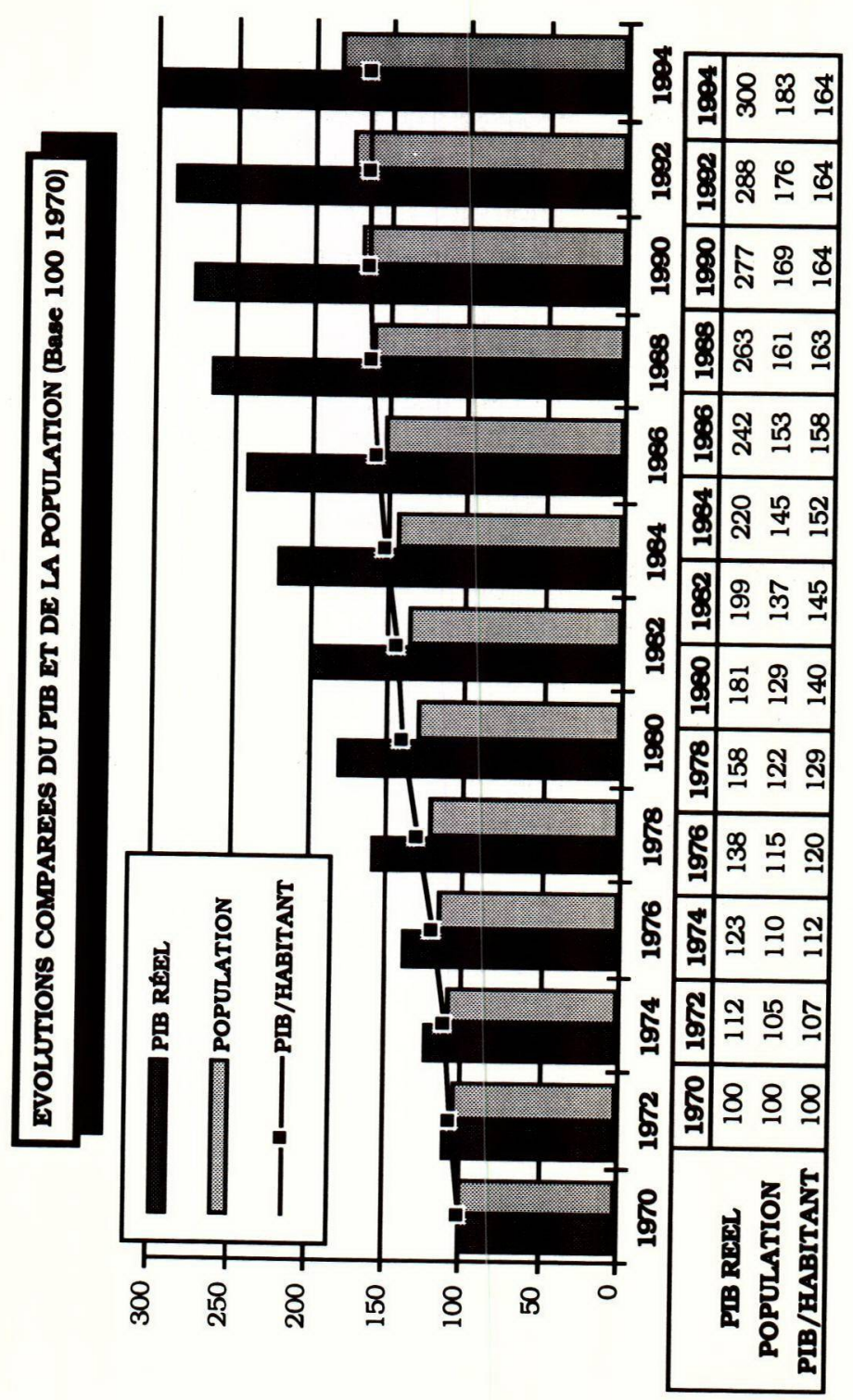

\section{Commentaires:}

De 1970 à 1986, le PIB réel de la Polynésie française a progressé à un rythme supérieur $\grave{a}+5 \%$ par an alors que la population augmentait au rythme de $+2,5 \%$ par an. Le produit intérieur brut moyen par habitant a pu ainsi régulièrement progresser sur cette période. 
- Depuis le milieu de la décennie 80 , le PIB réel ne progresse plus qu'à un rythme moyen de $+2 \%$ par an soit au même rythme que la population.

Le produit intérieur brut moyen par habitant est ainsi plafonné depuis presque 10 ans. Ce taux moyen inchangé cache une amélioration du pouvoir d'achat des populations disposant déjà d'un emploi et d'un logement équipé combinée à un développement rapide du chômage et de l'exclusion.

\section{PRINCIPAUX OBJECTIFS MACROECONOMIOUES DU PACTE A L'HORIZON} $\underline{2003}$

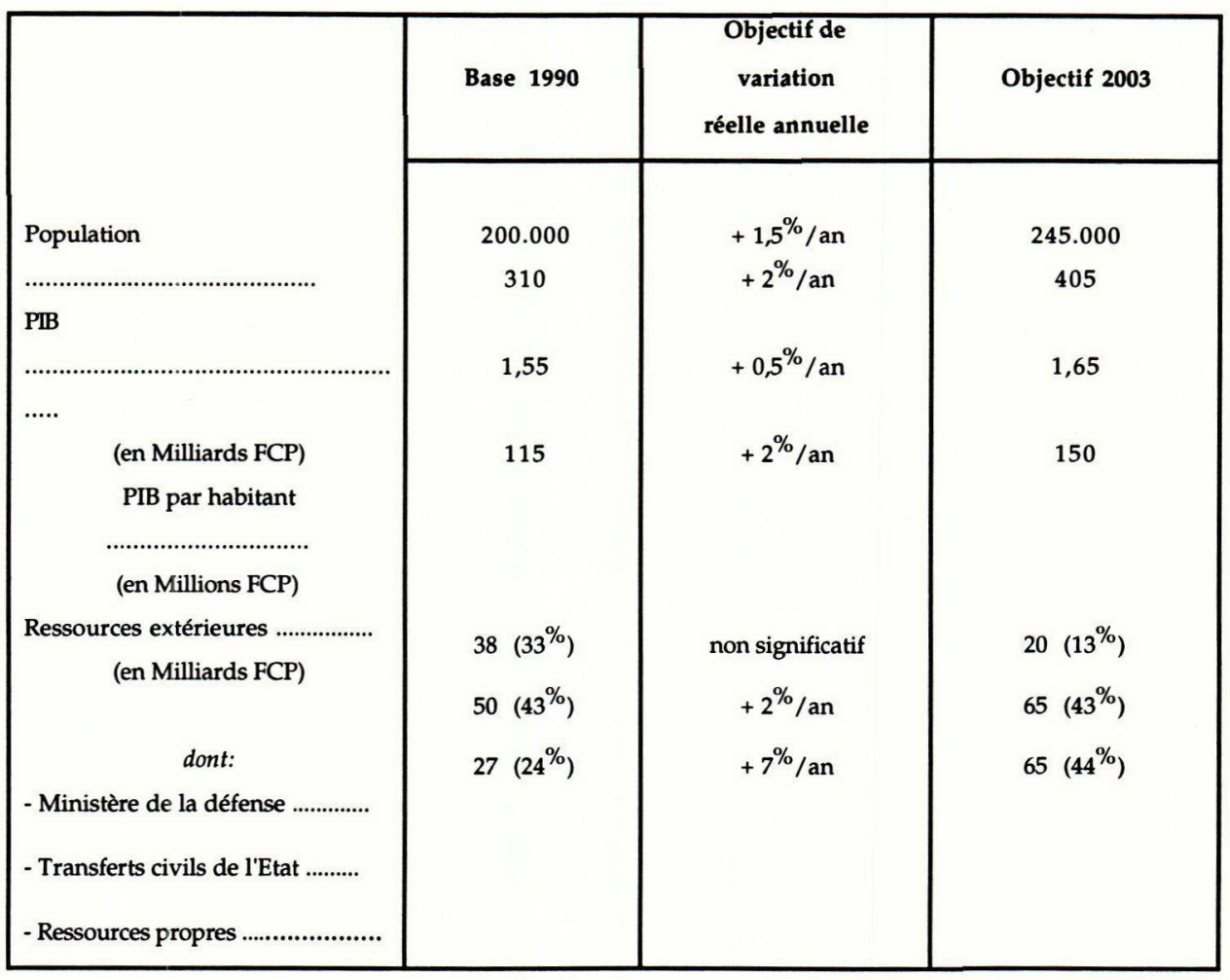

(Note IFF $=18,18$ FCP)

\section{Commentaires:}

- L'économie polynésienne est ouverte sur les échanges internationaux. L'évolution de son PIB depuis 30 ans a dépendu de l'évolution du volume des ressources extérieures et cette corrélation devrait rester vérifiée à l'avenir. 
- La variable déterminante de l'économie polynésienne est, en conséquence, le volume des ressources extérieures. Sur la décennie a venir, il est escompté une progression de ces ressources extérieures de $+2 \%$ par an permettant d'induire une croissance du PIB réel d'également $+2 \%$ par an.

- $\quad$ Il est pris pour hypothèse de travail que le Centre d'Expérimentation du Pacifique aura cessé ses activités en Polynésie française en 2003 d'où une division par deux des dépenses du ministère de la défense en Polynésie française.

- Pour conserver une croissance globale des ressources extérieures de $+2 \%$ par an malgré la disparition du CEP, il est indispensable qu'à la fois, les transferts civils de l'Etat progressent de $+2 \%$ par an et que le Territoire génère une progression de ses ressources propres au rythme de $+7 \%$ par an.

- Le PIB moyen par habitant ne progresserait que peu mais les différentes réformes structurelles (fiscalité - protection sociale...) devraient permettre une meilleure redistribution des richesses et une lutte contre la progression de l'exclusion sociale.

La réalisation des objectifs fondamentaux sur les 10 ans à venir suppose donc à la fois

- Une forte progression (rythme de $+7 \% / a n$ en réel) des ressources propres du Territoire de manière à réduire notre dépendance vis à vis de la Métropole en termes de balance des paiements de $75 \%$ à environ $50 \%$.

- D'importantes réformes structurelles sur le Territoire dans les domaines de la fiscalité, de la protection sociale et de son financement, des grilles salariales de la Fonction publique, du logement social... afin de mieux redistribuer les richesses et l'emploi et de réduire ainsi l'exclusion sociale.

Par une motion de soutien au projet de Pacte de progrès adoptée à la quasi unanimité le 15 janvier 1993, l'Assemblée Territoriale adoptait bien ainsi le schéma macroéconomique proposé et s'engageait à mettre en œuvre l'ensemble des réformes structurelles en matière économique et sociale comme le prouve le texte de cette motion ci-après:

\section{LES PROPOSITIONS DE LA DELEGATION POLYNESIENNE}

\section{La motion de soutien de l'Assemblée Territoriale}

\section{au projet du Pacte de progrès}

"L'Assemblée Territoriale réunie en session extraordinaire le 15 janvier 1993:

- $\quad$ Approuve et soutient le document présenté par la délégation polynésienne. Ce document élaboré conformément aux termes du protocole d'accord du 14 mai 1992 qui stipule: "les autorités du Territoire affirment clairement leur volonté de définir, en 
concertation avec l'Etat, un Pacte de progrès qui servira de base de discussion avec les représentants de l'Etat".

- Demande que soit validé par l'Etat le schéma de référence économique sur lequel est fondé le projet de Pacte et consistant à rééquilibrer l'économie du Territoire sur une période de 10 ans.

- $\quad$ Souhaite que les répartitions de financement du Pacte qui y sont prévues soient retenues par l'Etat; et que ce dernier s'engage clairement pour la part qui le concerne. L'Assemblée Territoriale quant à elle, s'engage de son côté à inscrire les crédits correspondants aux actions retenues dans les budgets à venir.

- $\quad$ S'engage à mettre en œuvre les réformes envisagées pour autant qu'elles sont de sa compétence et que l'Etat de son côté aura apporté, quand il y a lieu, la contribution juridique, technique ou financière qui lui incombe.

L'Assemblée Territoriale tient à cette occasion à réaffirmer sa volonté que la Polynésie française demeure un Territoire autonome au sein de la République. Elle rejette l'indépendance, comme l'administration directe par l'Etat, et considère que le statut d'autonomie est suffisamment évolutif pour être amélioré en fonction des nécessités de son développement."

Il est particulièrement intéressant de souligner ici que cette motion outre son soutien au projet de société économique et social développé dans le document intitulé Propositions de la délégation polynésienne pour le Pacte de progrès rappelait très clairement que pour l'ensemble des élus ayant voté cette motion, ce nouveau projet de société ne pouvait s'inscrire que dans un seul cadre statutaire, celui de Territoire d'outre-mer doté de l'Autonomie interne tout en rappelant que ce type de statut restait perfectible.

Peu de temps auparavant, les représentants de la société civile au Conseil économique, social et culturel avaient conclu de même leur rapport en écrivant:

\section{Extrait du Rapport du CESC sur le thème: \\ Quels choix pour l'avenir de la Polynésie}

"Parmi les différentes perspectives d'évolution institutionnelle de la Polynésie française, trois conceptions pourraient être envisagées:

1 L'indépendance, accession à la souveraineté, ne peut être que le fruit d'un long processus bien préparé et accepté par la plus grande majorité, faute de quoi et compte tenu de nombreuses faiblesses structurelles de notre pays, toute précipitation dans cette voie pourrait être dangeureuse. 
2 La départementalisation serait considérée comme un retour en arrière. Alors que même les collectivités métropolitaines bénéficient de plus d'autonomie, devenir un département après une période d'autonomie interne paraît à contre courant de l'histoire.

3 L'autonomie interne, au sein de la République, reste, malgré sa jeunesse relative et ses premiers échecs, un cadre institutionnel intéressant pour répondre aux aspirations qui semblent actuellement se dégager au sein de la population. Toutefois, les dysfonctionnements actuels appellent une refonte destinée à une clarification des textes, tant en matière de compétence, que de fonctionnement des institutions.

Quel que soit le choix retenu, il s'agit avant tout, pour la Polynésie française, de se forger un grand dessein et de le mettre en œuvre avec la Métropole dans un cadre institutionnel, économique, social et culturel cohérent et sans ambiguïté."

Il est, je pense clair, que cette option largement partagée pour le statut d'autonomie interne était assise sur le double objectif:

- De pouvoir s'administrer librement (d'où le rejet de la départementalisation) dans l'esprit de l'article 74 de la constitution.

- Tout en conservant des liens avec la Métropole pour préserver notamment la base de flux de transferts financiers (d'où le rejet de l'indépendance, du moins tant que notre économie restera aussi dépendante des transferts de l'Etat sans lesquels une faillite généralisée de l'économie polynésienne contemporaine serait induite). Cette position est moralement justifiée par les contributions apportées sans discontinuité par la Polynésie française à la défense de la Nation au travers de sa participation active aux deux grandes guerres et depuis 1964 par l'accueil du Centre d'Expérimentation du Pacifique.

Le Pacte de progrès devait donc être à la fois un pacte des polynésiens entre eux-mêmes pour mieux partager les richesses d'où la batterie de réformes proposées, mais aussi, bien évidemment, un pacte renouvellé entre la Polynésie française et l'Etat.

Dans le cadre du Pacte Etat-Territoire, la Polynésie française s'engageait pour sa part à développer ses ressources propres, à se réformer et l'Etat pour sa part devait définir la manière dont il accompagnerait la Polynésie française dans sa mutation profonde pour créer l'économie et la société de l'après CEP.

Il est important, à cet égard, de souligner combien les autorités de l'Etat et le législateur national ont tenu à respecter le statut dans l'élaboration du Pacte de progrès.

Ainsi, peut-on constater à la lecture de la loi d'orientation pour le dévelopement économique, social et culturel de la Polynésie française que le Parlement n'a pas défini mais 
a pris acte, en en approuvant l'esprit, des grandes orientations de politique économique, sociale et culturelle que les polynésiens ont eux-mêmes définis:

\section{Extrait de la loi d'orientation}

"L'Etat apportera notamment dans le cadre du contrat de développement et des conventions prévues à l'article 8 de la présente loi un appui technique et financier au territoire, afin d'aider ce dernier à atteindre les objectifs de développement économique, social et culturel que le territoire a définis dans l'exercice de ses compétences."

Le Texte de loi lui-même ne défini pas des orientations de politique mais précise comment sur des sujets particuliers, l'Etat entend accompagner le Territoire ou bien au travers d'une assistance technique, ou bien au travers d'aides financières ou d'adaptation de textes juridiques qui sont de son ressort.

Ainsi par exemple:

En matière de fiscalité, l'article 5 de la loi précise t'il:

"En matière de fiscalité, l'Etat apportera son concours technique à l'effort engagé en vue de moderniser les règles fiscales en vigueur dans le territoire. Il accroîtra le nombre des fonctionnaires détachés ou mis à disposition des services fiscaux et affectés au service des douanes du territoire.

L'Etat proposera d'autre part au territoire la conclusion d'une convention en vue de préciser les règles de territorialité de l'impôt et de prévenir la fraude fiscale."

En matière de protection sociale, les articles 3, 10 et 11 de la loi précisent-ils:

\section{Article 3}

"Dans le domaine de la santé publique et de la protection sociale, l'Etat apportera une assistance technique à la rénovation du système de santé et du régime de protection sociale du territoire. A cet effet, des experts seront mis à la disposition des autorités du territoire.

Les conditions d'attribution et d'utilisation des aides financières définies à l'article 10 et de l'assistance technique seront fixées par voie de convention.

L'Etat conclura avec le territoire de la Polynésie française un accord entre les régimes de protection sociale qui permettra la coordination de ces régimes pour l'ensemble des risques au profit des personnes assurées." 


\section{Article 10}

"En sus des contributions qu'il verse au titre de la solidarité, telles qu'elles sont fixées en 1993, l'Etat attribuera au régime de protection sociale de solidarité que le territoire s'engage à mettre en place une dotation de:

40 Millions de francs en 1994.

60 Millions de francs en 1995 ;

80 Millions de francs en 1996 ;

100 Millions de francs en 1997 ;

120 Millions de francs en 1998.

En sus de sa participation aux actions du territoire en matière de santé publique, telle qu'elle est fixée en 1993, l'Etat apportera à ces actions une contribution de:

1,8 Million de francs en 1994 ;

3,6 Millions de francs en 1995 ;

5,4 Millions de francs en 1996 ;

7,2 Millions de francs en 1997 ;

9 Millions de francs en 1998.

Les modalités des participations visées ci-dessus seront arrêtées dans une convention entre l'Etat et le territoire qui précisera les règles permettant le bon usage des fonds alloués."

\section{Article 11}

"Les personnes relevant du code des pensions civiles et militaires de retraite, qui exercent leurs fonctions en Polynésie française ou qui y résident en qualité de pensionnés au titre de ce code, sont affiliées à compter du $1^{\mathrm{er}}$ janvier 1995, pour les prestations en nature relevant de l'assurance maladie-maternité, au régime de sécurité sociale qui leur serait applicable si elles exerçaient leurs fonctions en métropole ou y résidaient en qualité de pensionnés au titre dudit code."

Note: L'article 11 s'applique aux fonctionnaires d'Etat qui sont de compétence Etat, statut article 3 - alinéa 14.

Il est clair, à la lecture de ces articles, qu'il appartient au Territoire et à lui seul de définir sa politique fiscale, sa politique de protection sociale et que l'Etat pour sa part 
après avoir approuvé les orientations décidées par le Territoire apporte son concours technique, juridique et financier pour aider à la réalisation de ces dernières.

De la même manière, le contrat de développement que beaucoup assimilent à tort avec le Pacte de progrès dont il ne constitue qu'une "pièce du puzzle" précise comment l'Etat aide le Territoire à engager la politique de développement de ses ressources propres, la politique d'équipements publics et de développement des archipels.

Il ressort de ces éléments que les différents textes constitutifs de l'édifice Pacte de progrès à savoir, les propositions de la délégation polynésienne pour le Pacte de progrès, les différentes délibérations de l'Assemblée Territoriale, la loi d'orientation, le contrat de développement, le contrat de ville, les différentes conventions Etat-Territoire (avec notamment le ministère de la défense, le ministère de la solidarité, le ministère de la culture...) ont tous scrupuleusement respecté l'esprit et la lettre du statut.

Comme nous n'avons cité pour le moment que des domaines de compétence territoriale, on peut souligner que des domaines de compétence Etat (Article 3 du statut) ont fait l'objet de propositions également dans l'élaboration du Pacte de progrès mais en dehors des propositions de la délégation polynésienne et donc directement cette fois dans le cadre de la loi d'orientation comme l'illustrent par exemple les article suivants qui concernent des domaines de la compétence de l'Etat.

\section{- Justice -}

(Article 3 du statut - alinéas 11-12 et 13)

\section{Article 4}

"L'Etat apportera, selon des modalités définies par convention, une assistance technique, notamment par la mise à disposition d'experts, aux services de la protection judiciaire de la jeunesse de la Polynésie française.

L'Etat instituera une commission de conciliation obligatoire en matière foncière dont la composition, la compétence et les règles de fonctionnement seront définies par une loi ultérieure."

\section{- Communes -}

(Article $3 d u$ statut - alinéa 15)

\section{Article 6}

"Dans le domaine de l'administration communale, le personnel communal sera doté d'un statut adapté à la situation particulière des communes du territoire et notamment à leurs capacités budgétaires." 


\section{LE PACTE DE PROGRÈS: UN PROJET QUI RESTE A CONCRETISER}

On a tant parlé du Pacte de progrès qu'il appartient désormais de passer des écrits et de la parole aux actes.

Pour que se concrétisent la nouvelle économie et la nouvelle société polynésienne, des conditions déterminantes devront être respectées. Certaines d'entre elles mettent en jeu le statut.

Ces conditions sont les suivantes:

1. La réussite du plan de développement des ressources propres.

L'objectif est ambitieux, $+7 \%$ par an et une part des ressources propres dans les ressources globales portée de $24 \%$ en 1990 à $44 \%$ en 2003 . Mais 1994 affiche déjà une quote-part de $34 \%$ comme le démontrent le tableau et le graphique ci-après. 


\begin{tabular}{|c|c|c|c|c|c|c|c|c|c|}
\hline \multirow[b]{2}{*}{ (Chiffres en Milliards de $F C P$ ) } & \multicolumn{4}{|c|}{ Réel } & \multirow{2}{*}{\begin{tabular}{|c|} 
Réestimé \\
1994
\end{tabular}} & \multicolumn{4}{|c|}{ Budget } \\
\hline & 1989/90 & 1991 & 1992 & 1993 & & 1994/96 & $1997 / 99$ & 2000/02 & 2003 \\
\hline Transferts militaires & $\underline{38}$ & $\underline{38}$ & 36 & $\underline{35}$ & 35 & $\underline{35}$ & $\underline{35}$ & $\underline{28}$ & 20 \\
\hline Transferts civils & 50 & 44 & 46 & 48 & 50 & 54 & 58 & 62 & 65 \\
\hline . & 42 & 44 & 45 & 45,5 & 47 & 48 & 51 & 54 & 57 \\
\hline . & 0 & 0 & 0 & 1 & 3 & 5 & 7 & 8 & 8 \\
\hline . & 8 & 0 & 1 & 1,5 & 0 & 1 & 0 & 0 & 0 \\
\hline Transferts Etat & 88 & 82 & 82 & 83 & 85 & 89 & 93 & 90 & 85 \\
\hline Ressources propres & 27 & 29 & 31 & 37 & 44 & 41 & 47 & 57 & 65 \\
\hline & 17 & 17 & 17 & 19,5 & 22,5 & 21 & 24 & 30 & 35 \\
\hline . & 6 & 7 & 8 & 9 & 10 & 10 & 12 & 14 & 15 \\
\hline . & 4 & 5 & 6 & 8,5 & 11,5 & 10 & 11 & 13 & 15 \\
\hline Ressources extérieures & $\underline{115}$ & $\underline{111}$ & $\underline{113}$ & $\underline{120}$ & $\underline{129}$ & $\underline{130}$ & 140 & 147 & 150 \\
\hline \% RESSOURCES PROPRES & $23,5 \%$ & $26,1 \%$ & $27,4 \%$ & $30,8 \%$ & $34 \%$ & $31,5 \%$ & $33,6 \%$ & $38,8 \%$ & $43,3 \%$ \\
\hline
\end{tabular}




\section{VARIATIONS DES RESSOURCES EXTERIEURES/BASE 1990}

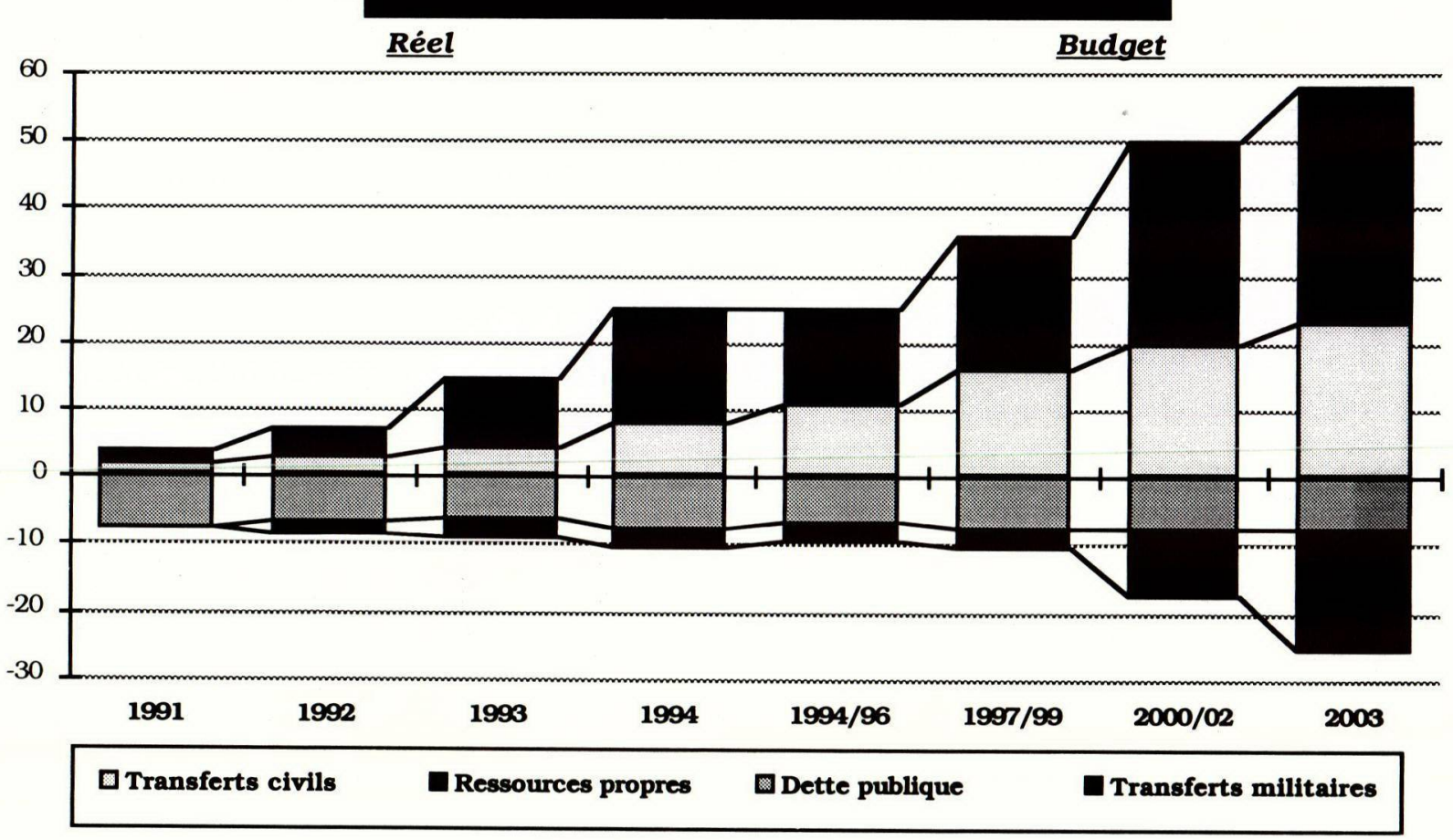




\section{Lé dégonflement de la Fonction publique.}

Les administrations pèsent trop dans l'économie polynésienne et doivent retrouver une place plus raisonnable dans la nouvelle économie à construire. Le ministère de la Défense nationale réduira très certainement ses activités et il faudra également que les administrations civiles territoriales et communales soient réduites tant en effectifs qu'en masse salariale pour réduire le poids de la pression fiscale nécessaire à son financement.

Cet effort majeur est engagé depuis peu d'années et devra être poursuivi.

\section{La mise en ouvre effective des grandes réformes structurelles.}

Les réformes majeures du système sont celles de la fiscalité et plus largement des mécanismes de prélèvements obligatoires ainsi que la réalisation de la protection sociale généralisée avec une remise à plat de la politique familiale.

4. La concrétisation des engagements financiers de l'Etat au titre du Pacte de progrès.

Si le respect des engagements de l'Etat au titre du Pacte de progrès sont loin de constituer une condition suffisante à la réussite du Pacte, il en constitue néanmoins une condition particulièrement nécessaire pour garantir les grands équilibres macroéconomiques du Territoire.

\section{Un climat politique et social relativement serein.}

Le développement ne peut se construire que sur la confiance. Aussi, un des risques majeurs qui pourrait remettre en cause la réalisation du Pacte de progrès est-il constitué par la menace d'une combinaison d'instabilité politique chronique et de troubles sociaux incontrôlés.

En développant les mécanismes de partage du pouvoir et de garantie du principe démocratique du respect du choix majoritaire, le statut pourrait jouer un rôle dans cette indispensable régulation des forces politiques et sociales.

Mais au-delà des textes les hommes au pouvoir qu'ils soient politiques ou syndicalistes devront apprendre à limiter leurs "chamailleries". Car, comme l'ont rappelé plusieurs intervenants, ce n'est en aucun cas le texte statutaire seul qui pourra définitivement rendre impossible tout dysfonctionnement des institutions.

Par ailleurs, il serait souhaitable pour le débat démocratique que les médias, en particulier la télévision, rééquilibrent significativement la part qu'ils consacrent aux véritables débats de société par rapport à l'espace réservé à la "politique spectacle". 


\section{Une régulation législative accompagnatrice de la mutation de la société.}

Nous l'avons vu, le Pacte de progrès ne pourra réussir que si des réformes structurelles majeures sont mises en œuvre dans des domaines aussi-divers que la politique de la famille, la politique foncière, la fiscalité, les mécanismes de protection sociale ...

Dans tous ces domaines, c'est la loi qui forge au fil des ans la structure économique et sociale d'une société.

Les rédacteurs de la constitution avaient, semble-t'il, clairement identifié que l'ensemble de l'Outre-Mer français était confronté à des environnements économiques, sociaux et culturels suffisamment distincts de la situation en Métropole pour justifier de l'application d'un environnement législatif et règlementaire différent et adapté aux réalités locales.

L'exemple contemporain le plus flagrant de situation fondamentalement distincte entre la Métropole et l'Outre-Mer est la question démographique.

Ainsi, il semble évident que la politique de la famille mise en œuvre en OutreMer ne devrait en aucun cas être calquée sur celle appliquée en Métropole puisque les objectifs (de régulation dans un cas et nataliste dans l'autre) sont fondamentalement opposés.

Il n'est pas inutile de rappeler à ce stade les articles de la constitution qui semblent établir ce "droit à la différence".

\section{Chapître: Des collectivités territoriales}

\section{Article 73}

"Le régime législatif et l'organisation administrative des départements d'OutreMer peuvent faire l'objet de mesures d'adaptation nécessitées par leur situation particulière".

\section{Article 74 (version 1958)}

"Les territoires d'Outre-Mer de la République ont une organisation particulière tenant compte de leurs intérêts propres dans l'ensemble des intérêts de la République. Cette organisation est définie et modifiée par la loi après consultation de l'assemblée territoriale intéressée". 


\section{Article 74 (version 1992)}

"Les territoires d'Outre-Mer de la République ont une organisation particulière tenant compte de leurs intérêts propres dans l'ensemble des intérêts de la République.

Les statuts des territoires d'Outre-Mer sont fixés par des lois organiques qui définissent, notamment, les compétences de leurs institutions propres, et modifiés, dans la même forme, après consultation de l'Assemblée Territoriale intéressée.

Les autres modalités de leur organisation particulière sont définies et modifiées par la loi après consultation de l'Assemblée Territoriale intéressée."

Pour tenter de comprendre ce que pourrait être l'esprit sous-jacent dans le statut d'Autonomie interne de la Polynésie française à la lumière du texte de la constitution, il est frappant de relire les articles 77 et 78 de cette dernière dans le chapître de la Communauté :

\section{Article 77}

"Dans la communauté instituée par la présente Constitution, les Etats jouissent de l'autonomie ; ils s'administrent eux-mêmes et gèrent démocratiquement et librement leurs propres affaires.

Il n'existe qu'une citoyenneté de la Communauté.

Tous les citoyens sont égaux en droit, quelles que soient leur origine, leur race et leur religion. Ils ont les mêmes devoirs".

\section{Article 78}

"Le domaine de la compétence de la Communauté comprend la politique étrangère, la défense, la monnaie, la politique économique et financière commune ainsi que la politique des matières stratégiques.

Il comprend en outre, sauf accord particulier, le contrôle de la justice, l'enseignement supérieur, l'organisation générale des transports extérieurs et communs et des télécommunications.

Des accords particuliers peuvent créer d'autres compétences communes ou régler tout transfert de compétence de la Communauté à l'un des ses membres".

On constate ainsi que la distribution des compétences entre l'Etat Français et le territoire de la Polynésie française est aujourd'hui quasiment celle prévue par l'article 78 de la constitution qui traite non pas des territoires d'Outre-Mer mais de la Communauté qui n'a jamais vu le jour dans les faits. 
Dans ce cadre de répartition des compétences, l'article 77 pose le principe de l'administration autonome dans l'ensemble des matières qui ne sont pas de la compétence de la Communauté.

L'article 77 précise clairement, par ailleurs, qu'il n'existe qu'une citoyenneté de la communauté et que: tous les citoyens sont égaux en droits et qu'ils ont les mêmes devoirs".

La difficulté fondamentale pour les rédacteurs de la constitution était de trouver une rédaction qui réussisse à rendre compatibles le respect du principe d'égalité des citoyens avec la reconnaissance d'une "spécifité législative" pour les collectivés d'Outre-Mer conçue, par conséquent, comme positive (permettant d'intégrer les différences) et non comme restrictive ( création d'une catégorie de sous-citoyens).

Or, l'expérience présente semble démontrer que tant pour les départements d'Outre-Mer que pour les territoires d'Outre-Mer, les rédacteurs de la constitution n'ont pas réussi à exprimer suffisamment clairement leur conception de l'équilibre à trouver entre le principe d'égalité et la reconnaissance des spécificités.

Notamment, comme l'a démontré Maître Moyrand hier, dans le cas particulier du statut d'Autonomie interne appliqué à la Polynésie française, on se trouve confronté à la contradiction fondamentale suivante qui est que de par le statut, un grand nombre de compétences traditionnellement dévolues au législateur national sont transférées au Territoire alors même que l'Assemblée Territoriale ne se voit reconnaître que le droit d'adopter des délibérations ayant valeur d'actes règlementaires et non de valeur législative.

Aussi, ces délibérations doivent-elles pour être légales être en conformité non seulement avec les principes constitutionnels mais également avec l'ensemble des principes généraux du droit.

Il est clair que le cadre normatif constitué par l'addition de la constitution, des lois et de la jurisprudence est par un mécanisme itératif le reflet d'un schéma de société donné.

On peut craindre, en conséquence, que toute volonté politique consistant à développer des politiques distinctes de celles menées en Métropole dans des matières aussi diverses que le droit foncier, la fiscalité ou la politique de la famille soit rendue aléatoire tant le texte constitutionnel laisse de marge d'interprétation possible aux magistrats du tribunal administratif, du conseil d'Etat et du conseil constitutionnel.

Or, le Pacte de progrès est précisément un projet de société bâti sur des propositions de politiques foncières, fiscales, sociales, familiales... qui se veulent délibérément différentes de celles menées en Métropole. 
Je souhaite insister particulièrement sur le fait qu'en faisant ce constat, ce n'est pas l'institution du tribunal administratif qui est mise en cause et encore moins les juges qui y exercent mais l'insuffisante clarté du texte constitutionnel.

En conclusion, il m'apparaît indispensable que le débat sur ce sujet soit porté bien audelà du seul statut de la Polynésie française à l'échelle de la constitution et pour l'ensemble de l'Outre-Mer français.

\section{BIBLIOGRAPHIE}

- Rapports de la $110^{\text {ème }}$ session régionale de l'Institut des hautes études de défense nationale à Papeete.

Juin 1992

- Etats Généraux de la Charte de Développement: Conclusion des ateliers de réflexion

Octobre 1992

- $\quad$ Rapport du Conseil Economique, Social et Culturel de Polynésie française sur le thème: "Quels choix pour l'avenir de la Polynésie française" Novembre 1992

- Pacte de Progrès économique, social et culturel de la Polynésie française, Propositions de la Délégation polynésienne Janvier 1993

- Propositions de l'Etat pour le Pacte de Progrès économique, social et culturel de la Polynésie français

Janvier 1993

- Avis du Conseil Economique et Social sur le projet de loi d'orientation pour le développement économique, social et culturel de la Polynésie française

Novembre 1993

- Loi d'orientation n 94-99 du 5 février 1994 pour le développement économique, social et culturel de la Polynésie française

\section{Pact of Progress for French Polynesia}

The expression Pact for Progress has been used extensively in French Polynesia since 1992 without its exact content being clearly identified. Thus, from a political point of view, the Pact for Progress is today for some the sole hope for the future well-being of French Polynesia and is for others a dead letter.

Three possible definitions of the Pact for Progress can be identified. They are:

- a turning point: The Pact for Progress can be analysed as a turning point in relation to the underlying spirit of the status of French Polynesia which recognises that fundamentally it is for the Polynesian population and their elected representatives to 
define the social, economic, and cultural environment in which they wish the development of the Territory to take place. The importance of what was at stake for the Territory and the unanimous and powerful support of lively forces that were brought to bear in this matter were the critical elements for its cohesion. French Polynesia experienced in the years 1991-1992 a particularly intense period of reflection and questioning by all the social and political forces of the Territory. Various studies had also provided a particularly clear diagnosis of the strengths and weaknesses of the Polynesian economy and society and it is on the basis of this that the key points for development and reform were established. The outcome of this period of reflection was the production in December 1992 of a document entitled "Proposals of the Polynesian Delegation for a Pact for Progress".

- a social project: The major factor in the diagnosis for the Pact for Progress was that French Polynesia had been the beneficiary over 25 years of a system of economic development based on transfers from the (Pacific Testing Centre) CEP and even more so (as in the other overseas departments and territories of France) on the rent system created by transfers of State money principally in the form of the remuneration for civil servants which benefits from a big premium relative to metropolitan France. But the combination of a strong demographic increase and a slowing of economic growth resulting from a net reduction in the annual amounts of the State transfers has lead for almost ten years to a very rapid increase in unemployment which affects almost $20 \%$ of the population. In respect to this, the Pact for Progress amounts to a true social project for French Polynesia.

- a reform yet to be concretised: Within the framework of the Pact between the State and the Territory, French Polynesia undertakes on its side to develop its own resources and to undertake reforms, and the State for its part has undertaken to define the manner in which it will support French Polynesia in the profound social changes which will be undertaken to create the economy and society for the period after CEP. It is important from this point of view to emphasise how much the State authorities and the national legislature have done to respect the status of the Territory in the drawing up of the Pact for Progress. In order to concretise the economy in the new Polynesian society, several key conditions must be fulfilled: a reduction in the size of the public service; the putting in place of major structural reforms; the formalising of the financial undertakings of the State in the context of the Pact for Progress; a political and social climate which is relatively calm; legislative regulation to accompany the transformation of the society.

The Pact for Progress is indeed a social project built on the basis of propositions that are political, land-based, fiscal, social, and familial, and which are deliberately different from those followed in metropolitan France such that the discussion on the matter must be pursued well beyond a consideration simply of the status of French Polynesia but also, in 
the context of the Constitution of France, and taking into consideration all of the French overseas departments and territories. 